\title{
Análisis de factores de agresividad de microcarcinoma papilar de tiroides*
}

\author{
Drs. PATRICIO CABANÉ T. ${ }^{1}$, PATRICIO GAC E. ${ }^{1}$, Int. CHRISTIAN ESVEILE A. ${ }^{2}$, \\ CRISTINA FERNÁNDEZ F. ${ }^{3}$, CLAUDIO LIBERMAN G. ${ }^{4}$, Al. IGNACIO BOZA T. ${ }^{5}$
}

\author{
Departamento de Cirugía, Hospital Clínico. \\ Interno Facultad de Medicina. \\ Departamento de Anatomía Patológica. \\ 4 Sección Endrocrinología, Departamento de Medicina. \\ 5 Alumno Facultad de Medicina. \\ Universidad de Chile. \\ Santiago, Chile.
}

\begin{abstract}
\section{Features influencing aggressiveness of papillary thyroid microcarcinoma}

Background: The risk factors determining the aggressiveness of papillary thyroid microcarcinoma (PTMC) are not well known. Aim: To determine if tumor size, along with other features of the tumor, influences its prognosis. Patients and Methods: We analyzed the medical records of 147 patients (age range 16-92 years, 93\% women) at the Clinical Hospital of University of Chile who underwent thyroid surgery and in whom at least one focus of PTMC was found. We determined the association between different clinical characteristics and the presence of capsular invasion, lymph nodal extension or recurrence. Results: A tumor size over $5 \mathrm{~mm}$, a follicular subtype and being aged more than 45 years, were significantly associated with the presence of capsular invasion. The latter two variables were protective. In the multivariate analysis, only a tumor size over $5 \mathrm{~mm}$ was significantly associated with thyroid capsule involvement. Conclusions: A tumor size over $5 \mathrm{~mm}$ is associated with capsular invasion in PTMC.
\end{abstract}

Key words: Thyroid cancer, microcarcinoma, capsular invasion.

\section{Resumen}

Introducción: Los factores de riesgo que determinan una conducta agresiva de microcarcinoma papilar de tiroides (MCPT) no se conocen. Nuestra hipótesis es que el tamaño del tumor, posiblemente junto con otras características del cáncer puede influir en el pronóstico de esta patología. Material y Método: Se analizaron las historias clínicas de 147 pacientes que se sometieron en nuestro hospital a cirugía de tiroides y en los cuales se encontró al menos un foco de MCPT. Resultados: Se determinó la existencia de una correlación entre las diferentes características clínicas y la presencia de invasión capsular, la extensión ganglionar linfático o

*Recibido el 20 de agosto de 2012 y aceptado para publicación el 11 de noviembre de 2012.

Los autores no refieren conflictos de interés

Correspondencia: Dr. Patricio Cabané T.

Santos Dumont 999, Santiago, Chile.

patriciocabane@paratiroides.cl 
la recidiva. En el análisis univariado, el tamaño del tumor mayor de $5 \mathrm{~mm}$, se correlacionó significativamente con la presencia de invasión capsular $(\mathrm{p}<0,05)$. Entre las variables estudiadas, sólo un tamaño superior a 5 mm se asoció significativamente con el compromiso de la cápsula tiroidea en el análisis multivariado. Conclusiones: Aunque en general el MCPT se comportan con baja agresividad, se encontró que aquellos que son mayores de $5 \mathrm{~mm}$ a menudo tienen invasión capsular, que se ha relacionado con aumento de la agresividad y recidiva. Se recomienda un tratamiento orientado según la presencia de factores de riesgo como las que se describen aquí.

Palabras clave: Tiroides, carcinoma micropapilar, factores de riesgo.

\section{Introducción}

El cáncer de tiroides representa aproximadamente el $1 \%$ de los nuevos cánceres encontrados anualmente ${ }^{1}$ y su forma de presentación más frecuente es el carcinoma papilar de tiroides, significando alrededor del $85 \%$ de las malignidades de esta glándula endocrina ${ }^{2}$. El tamaño de las lesiones neoplásicas pesquisadas es cada vez menor; si el tumor mide menos de $1,0 \mathrm{~cm}$, la OMS ha determinado que esta se denomine como microcarcinoma papilar de tiroides (MCPT).

A pesar de que la gran mayoría de los MCPT tienen buen pronóstico ${ }^{3-6}$, la sobrevida a 10 años ha sido estimada en un $84 \%$ por Domínguez et $\mathrm{al}^{7}$, algunas de estas lesiones pueden tener un comportamiento agresivo, exhibiendo recurrencias locorregionales $^{8,9}$, metástasis a linfonodos cervicales ${ }^{3,8,10,11}$ y progresión a la forma anaplásica de los carcinomas tiroideos ${ }^{12-14}$. Por otra parte, no hay consenso sobre cuáles son los factores de mal pronóstico para un $\mathrm{MCPT}^{15}$, proponiéndose entre ellos: el tamaño del tumor, la existencia de metástasis a linfonodos, edad mayor a 45 años, sexo masculino, cáncer no incidental, invasión extratiroidea, multifocalidad, enfermedad de Basedow Graves, entre otros ${ }^{3,16,17}$; tampoco hay acuerdo respecto al tratamiento óptimo de estas lesiones.

La presente investigación pretende determinar si el tamaño tumoral representa un mayor riesgo de agresividad en MCPT, analizando retrospectivamente 147 casos de pacientes con MCPT sometidos a cirugía tiroidea en el Hospital Clínico de la Universidad de Chile.

\section{Material y Método}

Se realizó un estudio de tipo retrospectivo a partir de biopsias de MCPT, donde se revisaron las fichas clínicas de 147 pacientes sometidos a tiroidectomía parcial o total entre 2000 y 2008 en nuestro hospital.

Los datos recolectados de la ficha de cada paciente fueron: sexo, edad, índice de masa corporal (IMC), lugar de residencia, presencia de antecedentes familiares y personales de patología tiroidea, existencia de comorbilidades en los pacientes, hábito tabáquico, función tiroidea, presentación clínica del carcinoma (síntomas de hiper/hipotiroidismo, masa cervical anterior visible y/o palpable, dolor cervical anterior, disfagia, disnea o disfonía), características de las ecografía tiroidea, resultado de la punción aspirativa con aguja fina (PAAF), tamaño tumoral, subtipo histológico del MCPT, multifocalidad, multicentricidad, compromiso capsular, extensión a linfonodos regionales o tejido extratiroideo, existencia de tiroiditis de Hashimoto o hiperplasia nodular en la biopsia diferida, complicaciones postquirúrgicas y recidiva. El punto de corte para definir hipocalcemia se determinó bajo los $8 \mathrm{mg} / \mathrm{dL}$ de calcio total.

La asociación entre algún signo de agresividad y otros factores clínicos se determinó usando la prueba $\chi^{2}$ de Pearson o la prueba exacta de Fisher, según correspondiera. El análisis multivariado para averiguar la importancia relativa de distintos factores que pudieran determinar mayor riesgo de agresividad (evidenciado por compromiso capsular, compromiso linfonodal o recidiva), se realizó utilizando regresión logística no condicionada. Se consideró como significativo un resultado con un $p<0,05$, asumiendo un intervalo de confianza de $95 \%$. La tabulación de los datos se realizó en el programa FileMaker 8.5; y el análisis estadístico de los mismos se efectuó con STATA 9.1 (StataCorp; TX, USA).

\section{Resultados}

\section{Características basales}

La edad de los pacientes al momento de la cirugía fluctuó entre 16 y 92 años (media: 45,56, mediana: 45,0 ); el 48,29\% de los pacientes tenía más de 45 años. El 92,5\% de los casos correspondió a mujeres y el $7,5 \%$ a hombres. El IMC promedio del grupo estudiado fue de $26,9 \mathrm{~kg} / \mathrm{m}^{2}$. El $87 \%$ provenía de la región Metropolitana de Santiago, lugar de emplazamiento de nuestro centro hospitalario.

Noventa y un pacientes $(61,9 \%)$ tenían alguna comorbilidad; siendo la hipertensión arterial la más frecuente, seguida por la obesidad. Un 39,45\% de los pacientes era fumador activo. Al momento del diagnóstico, 14 personas $(9,52 \%)$ tomaban regularmente levotiroxina vía oral. Un $8,84 \%$ de 
los casos tenía antecedentes familiares de patología tiroidea, predominando las neoplasias $(58,3 \%)$ por sobre las enfermedades benignas $(41,7 \%)$. Adicionalmente, 32 individuos $(21,76 \%)$ tenían historia previa de alteraciones benignas del parénquima o la función tiroidea. Clínicamente, 93,88\% de los pacientes se presentó sintomático en la primera consulta; en su mayoría, aumento de volumen cervical anterior y síntomas de hipertiroidismo.

Respecto al estado funcional de la tiroides, sólo hubo datos fidedignamente consignados en la ficha clínica de 73 pacientes; de los cuales $55(75,34 \%)$ eran eutiroideos, $11(15,06 \%)$ cursaban con hipertiroidismo y el resto $(9,58 \%)$ estaban en hipotiroidismo. La mayoría de los pacientes tenía un patrón multinodular a la ecografía $(55,10) ; 36,05 \%$ tenía un nódulo mayor a $15 \mathrm{~mm}$ de diámetro. Sesenta y seis pacientes $(44,89 \%)$ fueron estudiados con punción aspirativa con aguja fina (PAAF), siendo esta positiva para células neoplásicas en el 48,48\% de los casos, categoría VI, maligna según el sistema BETHESDA; es decir, sólo un $21,7 \%$ de los pacientes tuvieron un diagnóstico prequirúrgico de carcinoma papilar de tiroides (Tabla 1).

\section{Características de la cirugía}

La técnica quirúrgica preferida fue la tiroidectomía total en un $91,83 \%$, asociada a resección linfonodal en 12 pacientes $(8,88 \%)$; en 4 de 12 casos se realizó linfadenectomía del compartimiento central; en 2 , de los laterales; y en 6, de ambos compartimientos. La complicación postquirúrgica más frecuente fue la hipocalcemia, observada en un $63,94 \%$ de los casos, seguida por disfonía en el 10,88\% de los pacientes. Sesenta sujetos $(40,81 \%)$ fueron sometidos a aplicación postquirúrgica de yodo radiactivo. Cuatro pacientes $(2,72 \%)$ presentaron recidiva y debieron ser reoperados, a pesar de que 2 de ellos habían recibido una dosis ablativa de I-131 (Tabla 2).

\section{Características histológicas}

El tamaño tumoral promedio fue de 4,26 $\pm 2,65 \mathrm{~mm}$ (mediana: 4), y un $32,65 \%$ de las muestras demostró tumores mayores a $5 \mathrm{~mm}$. El subtipo histológico encontrado con más frecuencia fue el papilar $(61,22 \%)$, seguido por el folicular y luego, por el mixto. Un $15,64 \%$ de las biopsias mostraron compromiso capsular, un 26,53\% extensión
Tabla 1. Características basales de los pacientes estudiados

\begin{tabular}{|c|c|}
\hline Características basales & $\begin{array}{c}\text { Resultados } \\
\text { obtenidos }\end{array}$ \\
\hline $\begin{array}{l}\text { Edad promedio - años } \\
\text { Mediana } \\
\text { Mayores de } 45 \text { años }\end{array}$ & $\begin{array}{c}45,56 \pm 12,53 \\
45 \\
71(48,29)\end{array}$ \\
\hline Sexo masculino - $\mathrm{n}(\%)$ & $11(7,48)$ \\
\hline IMC & $26,96 \pm 4,93$ \\
\hline $\begin{array}{l}\text { Lugar de residencia - n }(\%) \\
\text { Región Metropolitana } \\
\text { Otras regiones de Chile }\end{array}$ & $\begin{array}{r}128(87,07) \\
19(12,92)\end{array}$ \\
\hline $\begin{array}{l}\text { Comorbilidades - n }(\%) \\
\text { Hipertensión arterial } \\
\text { Obesidad } \\
\text { Diabetes mellitus } \\
\text { Tabaquismo }\end{array}$ & $\begin{array}{rr}91 & (61,9) \\
41 & (27,89) \\
20 & (13,6) \\
7 & (4,76) \\
58 & (39,45)\end{array}$ \\
\hline $\begin{array}{l}\text { Antecedentes de enfermedades tiroi } \\
\text { Personales } \\
\text { Familiares }\end{array}$ & $\begin{array}{l}32(21,76) \\
13 \quad(8,84)\end{array}$ \\
\hline $\begin{array}{l}\text { Estado funcional tiroideo conocido - } \\
\text { Eutiroideo } \\
\text { Hipotiroidismo } \\
\text { Hipertiroidismo }\end{array}$ & $\begin{array}{r}55(75,34) \\
7 \quad(9,58) \\
11(15,06)\end{array}$ \\
\hline Uso de levotiroxina - $\mathrm{n}(\%)$ & $14(9,52)$ \\
\hline $\begin{array}{l}\text { Síntomas de presentación - n (\%) } \\
\text { Asintomático } \\
\text { Aumento de volumen cervical } \\
\text { Síntomas hipertiroidismo } \\
\text { Síntomas hipotiroidismo } \\
\text { Dolor } \\
\text { Disfagia } \\
\text { Disnea } \\
\text { Disfonía }\end{array}$ & $\begin{array}{rr}9 & (6,12) \\
62 & (42,17) \\
18 & (12,24) \\
15 & (10,2) \\
15 & (10,2) \\
12 & (8,16) \\
8 & (5,44) \\
8 & (5,44)\end{array}$ \\
\hline $\begin{array}{l}\text { Ecografía tiroidea - } \mathrm{n}(\%) \\
\text { Bocio uninodular } \\
\text { Bocio multinodular } \\
\text { Nódulo }>15 \mathrm{~mm}\end{array}$ & $\begin{array}{l}43(29,25) \\
81(55,10) \\
53(36,05)\end{array}$ \\
\hline $\begin{array}{l}\text { PAAF realizada - } \mathrm{n}(\%) \\
\text { Positiva para células neoplásicas, } \\
\text { grupo VI BETHESDA }\end{array}$ & $\begin{array}{l}66(44,89) \\
32(48,48)\end{array}$ \\
\hline
\end{tabular}

multifocal, y un 20,40\% multicentricidad. No se demostró permeación vascular en ningún especimen biopsiado. Once de doce de los pacientes a quienes se les resecó linfonodos, tenían alguno de ellos con células neoplásicas. La alteración histológica concomitante más frecuentemente encontrada fue la hiperplasia nodular $(43,53 \%)$, seguida por la tiroiditis de Hashimoto (34,69\%) (Tabla 3).

\section{Análisis univariado}

El análisis univariado mostró que el tamaño tumoral mayor a $5 \mathrm{~mm}$, el subtipo folicular y tener más de 45 años 
Tabla 2. Características quirúrgicas de los sujetos estudiados

\begin{tabular}{|lr|}
\hline Características de la cirugía & Resultados \\
Cirugía realizada - $\mathrm{n}(\%)$ & \\
Tiroidectomía subtotal & $12(8,16)$ \\
Tiroidectomía total & $135(91,83)$ \\
Con disección linfonodal $(\mathrm{n}=135)$ & $12(8,88)$ \\
$\quad$ Centrales & 4 \\
Laterales & 2 \\
Centrales y laterales & 6 \\
Calcemia postquirúrgica & $7,98 \pm 0,61$ \\
Promedio & 8,0 \\
Mediana & $102(69,38)$ \\
Complicaciones postquirúrgicas - n $(\%)$ & $94(63,94)$ \\
Hipocalcemia & $17(11,56)$ \\
Hipocalcemia sintomática & $16(10,88)$ \\
Disfonía & $4(2,72)$ \\
Disfagia & $60(40,81)$ \\
Aplicación de I-131 - n (\%) & $4(2,72)$ \\
\hline Recidiva - n (\%)
\end{tabular}

Tabla 4. Análisis univariado para el compromiso tumoral de la cápsula

\begin{tabular}{|c|c|c|}
\hline Variable & $\begin{array}{l}\text { Odds } \\
\text { ratio }\end{array}$ & $\begin{array}{c}95 \% \text { confidence } \\
\text { interval }\end{array}$ \\
\hline Papilar & 1,9794 & $0,6818-6,5349$ \\
\hline Folicular & 0,1111 & $0,0026-0,7482$ \\
\hline Mixto & 2,0185 & $0,5078-6,7952$ \\
\hline Lesión $>5 \mathrm{~mm}$ & 6,5714 & $2,2732-20,3988$ \\
\hline Multicentricidad & 1,4705 & $0,4270-4,4478$ \\
\hline Multifocalidad & 2,5198 & $0,8831-6,9462$ \\
\hline Tiroiditis de Hashimoto & 1,5572 & $0,5582-4,2076$ \\
\hline Hiperplasia nodular & 0,4016 & $0,1220-1,1642$ \\
\hline Hombre & 0,5181 & $0,0114-4,0044$ \\
\hline Edad $>45$ años & 0,3203 & $0,0974-0,9297$ \\
\hline Nódulo ecográfico $>15 \mathrm{~mm}$ & 0,6930 & $0,1768-2,6458$ \\
\hline PAAF positiva & 1,08 & $0,2784-4,1813$ \\
\hline
\end{tabular}

de edad, se correlacionan significativamente con la presencia de invasión capsular; en los últimos dos casos, a modo de factor protector ( $\mathrm{p}<0,05$; Tabla 4). No se encontró asociación entre otras variables y la infiltración linfonodal o la recidiva del tumor.

\section{Análisis multivariado}

El análisis multivariado de las variables subtipo histológico (papilar o folicular), tamaño tumoral mayor a $5 \mathrm{~mm}$, multicentricidad, multifocalidad,
Tabla 3. Características histológicas de las piezas quirúrgicas obtenidas

\begin{tabular}{|lc|}
\hline Características histológicas & Resultados \\
Subtipo histológico - n (\%) & \\
$\quad$ Papilar & $90(61,22)$ \\
Folicular & $37(25,17)$ \\
Mixto & $20(13,60)$ \\
Tamaño tumoral & \\
$\quad$ Promedio & $4,26 \pm 2,65$ \\
Mediana & 4,0 \\
Lesión > 5 mm. - n (\%) & $48(32,65)$ \\
Compromiso capsular - No $(\%)$ & $23(15,64)$ \\
Multifocalidad - n (\%) & $39(26,53)$ \\
Multicentricidad - n $(\%)$ & $30(20,40)$ \\
Compromiso linfonodal $(\mathrm{n}=12)$ & 11 \\
\hline Anomalías concomitantes - $\mathrm{n}(\%)$ & \\
Hiperplasia nodular & $64(43,53)$ \\
Tiroiditis de Hashimoto & $51(34,69)$ \\
\hline
\end{tabular}

Tabla 5. Análisis multivariado para el compromiso tumoral de la cápsula

\begin{tabular}{|lccr|}
\hline Variable & OR & $\mathbf{9 5 \%}$ CI & p-value \\
Papilar & 0,7967 & $0,1881-3,3736$ & 0,758 \\
Folicular & 0,0823 & $0,0067-1,0070$ & 0,051 \\
Lesión > $5 \mathrm{~mm}$ & 9,7271 & $3,1018-30,4641$ & $<0,001$ \\
Multicentricidad & 0,4067 & $0,1018-1,6242$ & 0,203 \\
Multifocalidad & 2,2424 & $0,7490-6,7131$ & 0,149 \\
T. Hashimoto & 1,7242 & $0,5382-5,5231$ & 0,359 \\
Hiperplasia nodular & 1,0012 & $0,3091-3,2424$ & 0,998 \\
Hombre & 0,3998 & $0,0401-3,9814$ & 0,434 \\
Edad $>45$ años & 0,6177 & $0,1986-1,9213$ & 0,405 \\
\hline
\end{tabular}

tiroiditis de Hashimoto, hiperplasia nodular, sexo masculino y edad mayor a 45 años; determinó que el único factor de riesgo para presentar compromiso capsular es tener una lesión neoplásica mayor a $5 \mathrm{~mm}(\mathrm{p}<0,05$; Tabla 5). No se pudo establecer otro tipo de asociación entre las variables arriba mencionadas y los indicadores de agresividad, compromiso linfonodal y recidiva.

\section{Discusión}

Existe controversia en cuanto al manejo que debe realizarse con un paciente con MCPT. Algunos son partidarios de una observación sistemática del ti- 
roides con controles temporalmente regulares ${ }^{13,14,18}$, mientras que otros prefieren la cirugía como vía terapéutica, ya sea mediante lobectomía/istmectomía ${ }^{19-21}$ o tiroidectomía total/casi-total ${ }^{3,8,15,16}$.

Clásicamente, los factores de riesgo de comportamiento agresivo de un carcinoma papilar de tiroides han sido el sexo masculino, edad mayor a 45 años, tamaño tumoral, el compromiso capsular y la invasión linfonodal, vascular o neural ${ }^{3,16,17}$. A pesar de que se ha insistido que lesiones menores de un centímetro (los MCPT) tendrían menor riesgo de recidiva y un comportamiento biológico menos agresivo con una baja tasa de metástasis a distancia $(0-3 \%)^{3,8}$, escasa mortalidad y -la gran mayoría de las veces- asintomáticos, encontrándose incidentalmente durante cirugías tiroideas por otras causas o en autopsias ${ }^{3-6}$; hay evidencia clínica creciente que orienta a que estos tumores también tendrían factores de riesgo que los predisponen a exhibir mayor agresividad, con un rango de recurrencias locorregionales que va desde 0 a $11 \%$ a 10 años ${ }^{8-9}$, metástasis a linfonodos cervicales $(8,6-50,5 \%)^{3,8,10-11}$ y progresión a la letal forma anaplásica de los carcinomas tiroideos ${ }^{2-14}$.

En esta serie no logramos demostrar una asociación estadística entre los factores de agresividad y recidiva debido al número de pacientes analizados y al poco tiempo de seguimiento. Sin embargo, creemos demostrar que un tamaño tumoral mayor a $5 \mathrm{~mm}$ ya tiene significancia en el compromiso capsular de la tiroides, uno de los principales factores de riesgo de metástasis y recidiva como ha sido descrito en la literatura nacional ${ }^{7}$.

A pesar del bajo número de pacientes en que se realizó disección linfonodal junto a la tiroidectomía en nuestra serie, en más de un $90 \%$ de los casos se hallaron metástasis linfonodales regionales. Otros reportes tienen cifras similares de compromiso linfonodal en pacientes con MCPT, lo que hablaría de lo agresivo que pueden llegar a ser lesiones tumorales de menos de $1 \mathrm{~cm}$.

Adicionalmente, y aunque el número de casos es pequeño, encontramos igual cantidad de pacientes con recidiva tratados y no tratados con radioyodo; algo similar a lo encontrado por Ross et al $^{22}$, quien demostró que la terapia con I-131 no redujo las recurrencias en pacientes con patología multifocal o con linfonodos afectados; esto debiera ser estudiado por ensayos clínicos controlados y aleatorizados, a fin de establecer el verdadero valor de la terapia con radioyodo en este tipo de pacientes.

Por todo lo anterior, recomendamos realizar tiroidectomía total en todo paciente con diagnóstico de MCPT, evaluar el compromiso linfonodal mediante ecotomografía preoperatoria y realizar una cirugía orientada a la disección por compartimientos en los casos que se justifique. Creemos que no se debe considerar el MCPT como una entidad aparte del cáncer papilar de tiroides mayor a $1 \mathrm{~cm}$ y que se debe considerar realizar el tratamiento quirúrgico estándar basado en los hallazgos histológicos en tumores incluso de menos de $5 \mathrm{~mm}$.

Dado que el advenimiento y el mayor uso de nuevas y mejores técnicas diagnósticas como la ecografía de alta resolución y la biopsia por punción aspirativa con aguja fina (PAAF), garantiza que el tamaño de las lesiones neoplásicas pesquisadas es cada vez menor, y que existe evidencia de que mutaciones del gen BRAF, la sobreexpresión de las proteínas EGFR y COX-2 y las alteraciones en componentes de vías de transducción de señales

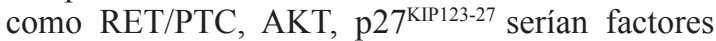
de riesgo de mayor agresividad de $\mathrm{MCPT}^{3,16,17,28-31}$; probablemente en el futuro debamos clasificar nuevamente estos casos del punto de vista molecular, teniendo certeza del comportamiento biológico de los distintos subtipos de cáncer de tiroides y no basarnos en un tamaño de corte arbitrario para definir riesgos para cada paciente.

\section{Referencias}

1. Jemal A, Tiwari RC, Murray T, Ghafoor A, Samuels A, Ward E, et al. Cancer statistics. CA Cancer J Clin. 2004;54:8-29.

2. Sugitani I, Kasai N, Fujimoto Y, Yanagisawa A. A novel classification system for patients with PTC: addition of the new variables of large ( $3 \mathrm{~cm}$ or greater) nodal metastases and reclassification during the follow-up period. Surgery 2004;135:139-48.

3. Sakorafas GH, Giotakis J, Stafyla V. Papillary thyroid microcarcinoma: A surgical perspective. Cancer Treatment Reviews 2005;31:423-38.

4. Fink A, Tomlinson G, Freeman JL, Rosen IB, Asa SL. Occult micropapillary carcinoma associated with benign follicular disease and unrelated thyroid neoplasms. Mod Pathol. 1996;9:816-20.

5. Harach HR, Franssila KO, Wasenius VM. Occult Papillary Carcinoma of the Thyroid. A "Normal" Finding in Finland. A Systemic Autopsy Study. Cancer 1985;56:531-8.

6. Baek SK, Jung KY, Kang SM, Kwon SY, Woo JS, Cho $\mathrm{SH}$, et al. Clinical Risk Factors Associated with Cervical Lymph Node Recurrence in Papillary Thyroid Carcinoma. Thyroid. 2010;20:147-52.

7. Domínguez M, Torres J, Pérez G, Volpato R, Jadresic A, López E. Estudio de los factores pronosticadores de la sobrevida del cáncer diferenciado del tiroides. Rev Chil Cir. 2011;63:566-72.

8. Pazaitou-Panayiotou K, Capezzone M, Pacini F. Clinical Features and Therapeutic Implication of Papillary Thyroid Microcarcinoma. Thyroid 2007;17:1085-92. 
9. Grant CS, Hay ID, Gough IR, Bergstralh EJ, Goellner $\mathrm{JR}$, McConahey WM. Local recurrence in papillary thyroid carcinoma: is extent of surgical resection important? Surgery 1988;104:956-62.

10. Chow SM, Law SC, Chan JK, Au SK, Yau S, Lau WH. Papillary microcarcinoma of the thyroid. Prognostic significance of lymph node metastasis and multifocality. Cancer 2003;98:31-40.

11. Baudin E, Travagli JP, Ropers J, Mancusi F, Bruno-Bossio G, Caillou B, et al. Microcarcinoma of the thyroid gland: the Gustave-Roussy Institute experience. Cancer 1998;83:553-9.

12. Trovisco V, Soares P, Preto A, Castro P, Valdemar M, Sobrinho-Simões M. Molecular genetics of papillary thyroid carcinoma - great expectations. Arq Bras Endocrinol Metab. 2007;51:643-53.

13. Santarpia L, El-Naggar AK, Cote GJ, Myers JN, Sherman SI. Phosphatidylinositol 3-Kinase/AKT and Ras/ Raf-Mitogen-Activated Protein Kinase Pathway Mutations in Anaplastic Thyroid Cancer. J Clin Endocrin Metab. 2008;93:278-84.

14. Gac P, Amat J, Cabané P. Carcinoma anaplástico de tiroides: ¿Indiferenciación de carcinoma papilar? Rev Chil Cir. 2006;58:77.

15. Pearce E, Braverman L. Papillary Thyroid Microcarcinoma Outcomes and Implications for Treatment. J Clin Endocrinol Metab. 2004;89:3710-2.

16. Pellegreti G, Scollo C, Lumera G, Regalbuto C, Vigneri R, Belfiore A. Clinical behavior and outcome of papillary thyroid cancers smaller than $1.5 \mathrm{~cm}$ in diameter: study of 299 cases. J Clin Endocrinol Metab. 2004;89:3713-20.

17. Lim DJ, Baek KH, Lee YS, Park WC, Kim MK, Kang MI, et al. Clinical, Histopathological, and Molecular Characteristics of Papillary Thyroid Microcarcinoma. Thyroid 2007; 17:883-8.

18. Ito Y, Uruno T, Nakano K, Takamura Y, Miya A, Kobayashi K, et al. An Observation Trial Without Surgical Treatment in Patients with Papillary Microcarcinoma of the Thyroid. Thyroid. 2003;13:381-7.

19. Miki H, Oshimo K, Inone H, Kawano M, Tanaka K, Komaki K, et al. Diagnosis and surgical treatment of small papillary carcinomas of the thyrois gland. J Surg
Oncol. 1993;54:78-80.

20. Rassael H, Thompson LD, Heffess CS. A rationale for conservative management of microscopic papillary carcinoma of the thyroid gland: a clinicopathologic correlation of 90 cases. Eur Arch Otorhinolaryngol. 1998; 225:462-7.

21. Cohn KH, Bäckdahl M, Forsslund G, Auer G, Zetterberg A, Lundell G, et al. Biologic considerations and operative strategy in papillary thyroid carcinoma: arguments against the routine performance of total thyroidectomy. Surgery 1984;96:957-71.

22. Ross DS, Litofsky D, Ain KB, Bigos T, Brierley JD, Cooper DS, et al. Recurrence After Treatment of Micropapillary Thyroid Cancer. Thyroid 2009;19:1043-8.

23. Ciampi R, Nikiforov YE. RET/PTC rearrangements and BRAF mutations in thyroid tumorigenesis. Endocrinology 2007;148:936-41.

24. Melillo RM, Castellone MD, Guarino V, De Falco V, Cirafici AM, Salvatore G, et al. The RET/PTC-RASBRAF linear signaling cascade mediates the motile and mitogenic phenotype of thyroid cancer cells. J Clin Invest. 2005;115:1068-81.

25. Patel KN, Singh B. Genetic Considerations in Thyroid Cancer. Cancer Control 2006;13:111-8.

26. Shinohara M, Chung YJ, Saji M, Ringel MD. AKT in Thyroid Tumorigenesis and Progression. Endocrinology 2007;148:942-7.

27. Manning BD, Cantley LC. AKT/PKB signaling: navigation downstream. Cell 2007;129:1261-74.

28. Trovisco V, Soares P, Sobrinho-Simões M. B-RAF mutations in the etiopathogenesis, diagnosis, and prognosis of thyroid carcinomas. Human Pathology 2006;37:7816.

29. Fugazzola L, Mannavola D, Cirello V, Vannucchi G, Muzza M, Vicentini L, et al. BRAF mutations in an Italian cohort of thyroid cancers. Clinical Endocrinology 2004;61:239-43.

30. Lee JH, Lee ES, Kim YS. Clinicopathologic Significance of BRAF V600E mutation in papillary carcinomas of the thyroid. Cancer 2007;110:38-46.

31. Xing M. BRAF mutation in papillary thyroid cancer: pathogenic role, molecular bases, and clinical implications. Endocrine Reviews 2007;28:742-62. 